\title{
The risk and outcome of cerebral oedema developing during diabetic ketoacidosis
}

\author{
J A Edge, M M Hawkins, D L Winter, D B Dunger
}

\begin{abstract}
Background-Cerebral oedema is a major cause of morbidity and mortality in children with insulin dependent diabetes. Aims-To determine the risk and outcome of cerebral oedema complicating diabetic ketoacidosis (DKA).

Methods-All cases of cerebral oedema in England, Scotland, and Wales were reported through the British Paediatric Surveillance Unit between October 1995 and September 1998. All episodes of DKA were reported by 225 paediatricians identified as involved in the care of children with diabetes through a separate reporting system between March 1996 and February 1998. Further information about presentation, management, and outcome was requested about the cases of cerebral oedema. The risk of cerebral oedema was investigated in relation to age, sex, seasonality, and whether diabetes was newly or previously diagnosed.
\end{abstract}

Results-A total of 34 cases of cerebral oedema and 2940 episodes of DKA were identified. The calculated risk of developing cerebral oedema was 6.8 per 1000 episodes of DKA. This was higher in new (11.9 per 1000 episodes) as opposed to established (3.8 per 1000) diabetes. There was no sex or age difference. Cerebral oedema was associated with a significant mortality (24\%) and morbidity (35\% of survivors).

Conclusions-This first large population based study of cerebral oedema complicating DKA has produced risk estimates which are more reliable and less susceptible to bias than those from previous studies. Our study indicates that cerebral oedema remains an important complication of DKA during childhood and is associated with significant morbidity and mortality. Little is known of the aetiology of cerebral oedema in this condition and we are currently undertaking a case control study to address this issue.

(Arch Dis Child 2001;85:16-22)

Keywords: diabetic ketoacidosis; cerebral oedema

Cerebral oedema has been recognised since the 1960 s as a devastating and unpredictable complication of diabetic ketoacidosis (DKA) in children, although the pathological features were first described in adults in $1936 .{ }^{1}$ The mortality from cerebral oedema has been reported to be as high as $90 \%,{ }^{2}$ although most series of case reports have suggested lower mortality figures of around $50-60 \% .^{3-5}$ In the
UK around $70-80 \%$ of diabetes related deaths in children under 12 years of age are caused by cerebral oedema. ${ }^{6}$

Survival from an episode of cerebral oedema complicating DKA may be associated with significant neurological sequelae. ${ }^{389}$ Thus the mortality and morbidity resulting from this complication of diabetes is of considerable importance. Yet the true risk of cerebral oedema has been difficult to estimate, as most previous studies have used retrospective surveys of DKA cases from single hospitals, ${ }^{458}$ which may be biased by unknown selection influences. In the USA, data from these analyses indicate that the likelihood of developing cerebral oedema during an episode of DKA is approximately $0.7-1.0 \% .{ }^{4}{ }^{10}$ However, the lack of a population base in these studies may result in ascertainment bias. Furthermore, therapeutic fluid regimens that have been implicated in the pathogenesis of cerebral oedema may differ between the USA and the UK.

In order to determine the risk of developing cerebral oedema, and its outcome during an episode of DKA, we have completed a national study in England, Scotland, and Wales. We report data from national surveillance for cases of cerebral oedema and an independent system of ascertainment of episodes of DKA.

\section{Methods}

SYSTEM OF ASCERTAINMENT OF CEREBRAL OEDEMA

Paediatricians were asked to report any cases of possible cerebral oedema, or any death, during the assessment or treatment of DKA, in children up to their 16th birthday through the British Paediatric Surveillance Unit (BPSU). The BPSU provides an active reporting system for the surveillance of certain rare childhood disorders. ${ }^{11}$ The Unit coordinates a reporting scheme whereby all consultant paediatricians in the UK are sent a monthly reporting card containing a list of specific conditions. The consultants are asked to report cases of conditions seen in the preceding month, or to tick a "nil return" box. On average $94 \%$ of BPSU clinicians return the report card each month.

The criteria for reporting a case for this study were as follows:

(1) Sudden or unexpected deterioration in conscious level in a child with diabetic ketoacidosis

(2) Any death during assessment or management of DKA.

Collection of cases ran for three years from October 1995 to September 1998 inclusive. When a case was reported to the BPSU, further details were requested from each clinician by questionnaire. A case of cerebral oedema was 
confirmed when a deterioration in the level of consciousness was accompanied by one or more further signs of raised intracranial pressure (hypertension and bradycardia with or without breathing pattern abnormalities, pupillary abnormalities, squint, blurred disc margins, decerebrate or decorticate posturing, respiratory arrest, or radiological evidence of moderate to severe cerebral oedema). A further questionnaire was sent 6-12 months after the episode to the paediatrician caring for each of the survivors of cerebral oedema, asking for details of the outcome, with respect to neurological function and schooling.

SYSTEM OF ASCERTAINMENT OF DIABETIC

KETOACIDOSIS

In order to ascertain all occurrences of DKA, irrespective of whether cerebral oedema had developed, a separate national reporting system was established and organised from Oxford. Paediatricians caring for children with diabetes were identified from several sources: a survey of paediatricians caring for children with diabetes carried out by the British Diabetic Association in $1994,{ }^{12}$ the British Paediatric Association handbook, ${ }^{13}$ a survey of hospitals providing educational facilities for children as inpatients, ${ }^{14}$ and the membership register of the British Society for Paediatric Endocrinology and Diabetes. Consultants at regional centres were also asked to provide details of the other paediatricians in the region with responsibility for children with diabetes. In this way, we aimed to achieve full coverage of all the hospitals which admit children with DKA in England, Scotland, and Wales.

A total of 299 paediatricians in England, Scotland, and Wales were thus identified and contacted. Fifty five consultants either did not look after children with diabetes, or worked in a hospital where one of the other paediatricians agreed to notify all the cases. Only one consultant approached declined to participate (but another consultant in the same hospital agreed to report). Thus 243 consultants in 231 hospitals agreed to carry out the monthly reporting of episodes of DKA for the two years between March 1996 and February 1998. The definition of DKA was "decompensated diabetes mellitus with evidence of ketoacidosis ( $\mathrm{pH}<7.3$ or plasma bicarbonate $<18 \mathrm{mmol} / 1$ or heavy ketonuria)". Data requested about each episode of DKA were: date of birth, sex, hospital number, admission date of episode, and whether the child had newly diagnosed ("new") or known ("old") diabetes.

\section{STATISTICAL METHODS}

To estimate the risk of cerebral oedema in DKA we restricted attention to the two year period for which both systems of ascertainment were running concurrently (March 1996 to February 1998). During this period it was possible to relate a numerator to an appropriate denominator. The two systems of ascertainment were established to run independently. In practice, it emerged that some paediatricians had considered it unnecessary to report patients who developed cerebral oedema (and who they therefore notified to the already established BPSU system) to the system established in Oxford to ascertain all occurrences of DKA irrespective of whether cerebral oedema developed. Such consultants regarded more than one notification as redundant. During the two year period there were four cases of cerebral oedema notified through the BPSU system which were not separately notified through the DKA system. The four consultants notifying these four cases of cerebral oedema had a good record of DKA notifications: of the total notification months accumulated between them $(4 \times 24=96)$, for only two of these months were notifications not received. Therefore we considered it acceptable to include such notifications received only through the BPSU in both the numerator and denominator in our risk calculations.

To assess whether the risk of developing cerebral oedema, during an episode of DKA, was related to sex, age, or seasonality, or whether diabetes was new or previously diagnosed, a linear logistic regression model was used. Sex and whether diabetes was old or new were each fitted as a dichotomous factor. Seasonality was also fitted as a factor with levels corresponding to each of the following periods: March-May, July-August, September-November, and December-February. To assess the possible effect of age, two factorisations were used: one comprised levels corresponding to $0-3,4-7$, $8-11$, and $12-15$ years; the alternative factorisation corresponded $0-2,3-5,6-8,9-11$, and $12-15$ years. The null hypothesis of homogeneity on the risk of cerebral oedema across different levels of age was tested against two alternative hypotheses, unspecified heterogeneity and linearity. Linearity was assessed in the standard way of assigning the values of the midpoint of each age range to a regression variate.

Patients may present with more than one episode of DKA and it may be hypothesised that the risk of cerebral oedema occurring within different episodes to a given patient may be more similar compared with the risk in other patients. There was no evidence of such overdispersion in these data and results are presented without an adjustment for such an effect.

The study was approved by the Central Oxford Research Ethics Committee, and by other local ethics committees where submission was considered necessary. There was no contact with patients or their families and the medical records of the patients were not examined.

\section{Results}

CASES OF CEREBRAL OEDEMA

A total of 126 returns were made to the BPSU between October 1995 and September 1998 (fig 1). Subsequently questionnaires were returned in 122 cases. Twenty six were "reporting errors" or "non-cases" and 30 were duplicate reports. There were 34 cases of cerebral oedema confirmed on the basis of these questionnaires ( 14 boys and 20 girls, age range $1-15$, median 11 years), of which eight resulted in death. 


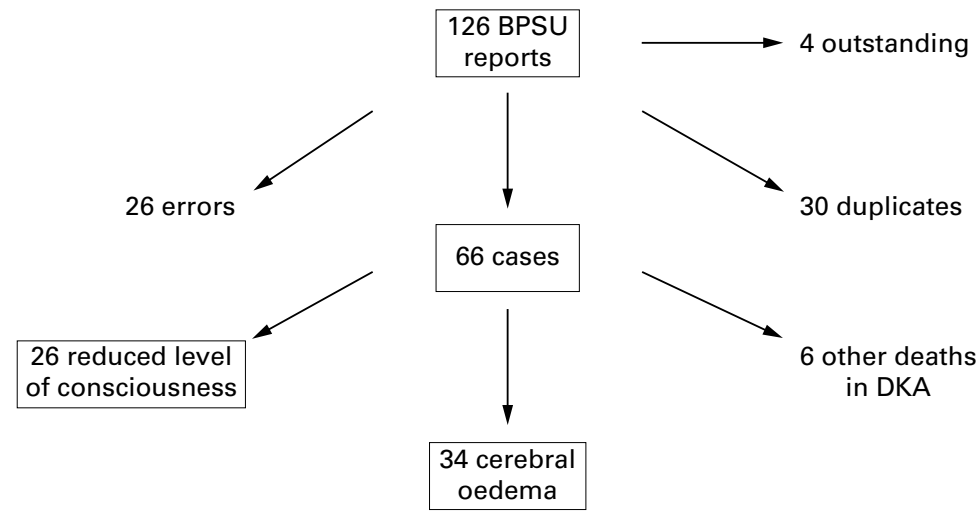

Figure 1 Returns to the BPSU between October 1995 and September 1998.

In addition there were 26 cases of unexplained deterioration in conscious level but without any other signs of raised intracranial pressure, and six deaths during ketoacidosis which were not associated with cerebral oedema occurring during hospital treatment of DKA. Two of the deaths may have been the result of cerebral oedema which occurred before admission to hospital, one leading to death at home and one leading to death some hours after admission. The cases of reduced conscious level alone and the six deaths not caused by cerebral oedema in hospital were not included in the analysis.

The 34 cases of cerebral oedema presented with a reduced level of consciousness and combinations of other signs of raised intracranial pressure: $14(41 \%)$ had hypertension and bradycardia, $12(35 \%)$ had pupil abnormalities, five $(15 \%)$ had decerebrate or decorticate posturing, and $10(29 \%)$ had a respiratory arrest. In 21 of the 34 cases, computed tomography (CT) scan confirmation of cerebral oedema was obtained; one further child had a normal CT scan but there were other features of cerebral oedema and the timing of the scan was unknown.

Eight of the children with cerebral oedema died (three boys, age $1-15$, median 7.5 years; $24 \%$ mortality). There was no difference in the age or sex distribution of those who died and survived. Of the 26 survivors, 17 children $(65 \%)$ have no obvious permanent sequelae (one child had temporary squint and ataxia which resolved over several months). The remaining nine children have a variety of persisting new problems following the episode of cerebral oedema, including motor deficits $(n=8)$, visual impairment $(n=6)$, short term memory loss $(n=6)$, speech problems such as dysphasia and dysarthria $(n=4)$, and convulsions $(n=2)$. These children also have a variety of learning and emotional problems, including poor concentration, disinhibited behaviour, and emotional lability. All 10 children who had a respiratory arrest during the development of cerebral oedema either died (all eight deaths were in this group) or had major neurological sequelae.

EPISODES OF DKA

Of a possible $5832(24 \times 243)$ monthly reports of episodes of DKA which could be expected,
5278 were returned $(90.5 \%$ complete reporting overall). There were 18 consultants who returned less than half of their 24 possible reports; these made up $64 \%$ of the total number of missing months. These 18 consultants were removed from the database and the reporting from the remaining 225 consultants was then $96.3 \%$ complete. The remainder of the results relate to these 225 consultants.

Complete data were available on 2940 episodes of DKA in 2052 patients. There were 1707 episodes in girls (1152 old and 555 new diabetes) and 1233 episodes in boys (693 old and 540 new diabetes). Of the known diabetics, 360 had more than one episode during the two year period and in these, the number of episodes ranged from two to 36 . One hundred patients with four or more episodes had 661 admissions; thus $4.8 \%$ of patients accounted for $22.5 \%$ of the total number of episodes of DKA reported.

\section{THE RISK OF CEREBRAL OEDEMA}

Twenty one cases of cerebral oedema were notified during the relevant two year period, but one case was notified by one of the 18 clinicians whose notifications of DKA may have been biased and so was excluded. Thus 20 cases of cerebral oedema were notified, complicating 2940 episodes of DKA. Therefore the overall risk of developing cerebral oedema was 6.8 per 1000 episodes of DKA. Table 1 presents variation of the risk of cerebral oedema by sex, age, new or old diabetes, and seasonality. There was no evidence that sex or seasonality was statistically significantly related to risk of cerebral oedema. There was a suggestion that the younger patients may be at greater risk, but this was not statistically significant, irrespective of whether age was considered at four or five levels and whether a linear or nonlinear relation with age was postulated. Newly diagnosed diabetes was associated with about three times the risk of cerebral oedema associated with previously diagnosed diabetes (odds ratio $3.2 ; 95 \%$ confidence interval 1.3 to 7.9 ; $\mathrm{p}=0.012)$.

\section{Discussion}

This is the first population based study of cerebral oedema. The overall risk of cerebral oedema was estimated to be 7 per 1000 episodes of DKA. The risk was 4 per 1000 in previously diagnosed diabetics and 12 per 1000 in those with newly diagnosed diabetes. Although there was no evidence that the risk of cerebral oedema was related to age, sex, or season of occurrence, there was a non-significant trend towards an increased risk in younger patients, who tend to be the most ill at diagnosis. ${ }^{15} \mathrm{~A}$ longer period of surveillance would be required to confirm this. Of the 126 reports to the BPSU, questionnaires were not returned on four; it is very unlikely that these would relate to any further cases of cerebral oedema as the consultants or junior staff involved could not recall any cases. Thus we are confident that we have discovered all possible cases of cerebral oedema during the period of the study. 
Table 1 Risk of developing cerebral oedema in relation to age, sex, season, and whether diabetes was newly diagnosed or already known

\begin{tabular}{|c|c|c|c|c|c|c|c|c|c|c|}
\hline \multirow[b]{3}{*}{$\begin{array}{l}\text { Classification } \\
\text { factor }\end{array}$} & \multirow[b]{3}{*}{ Levels of factor } & \multirow[b]{3}{*}{$\begin{array}{l}\text { Total } \\
\text { episodes of } \\
D K A\end{array}$} & \multirow[b]{3}{*}{$\begin{array}{l}\text { No. episodes of } \\
\text { DKA affected by } \\
\text { cerebral odema }\end{array}$} & \multirow[b]{3}{*}{$\begin{array}{l}\text { Risk } \\
\text { (per 1000) }\end{array}$} & \multicolumn{6}{|c|}{ Logistic regression analysis } \\
\hline & & & & & \multicolumn{3}{|c|}{ Unadjusted analysis } & \multicolumn{3}{|c|}{ Adjusted analysis* } \\
\hline & & & & & $\begin{array}{l}\text { Odds } \\
\text { ratios }\end{array}$ & $95 \% C I$ & $\begin{array}{l}\text { p value for the } \\
\text { likelihood ratio test of } \\
\text { homogeneity across } \\
\text { factor levels }\end{array}$ & $\begin{array}{l}\text { Odds } \\
\text { ratios }\end{array}$ & $95 \% C I$ & $\begin{array}{l}p \text { value for the } \\
\text { likelihood ratio test of } \\
\text { homogeneity across } \\
\text { factor levels }\end{array}$ \\
\hline Sex & $\begin{array}{l}\text { Female } \\
\text { Male }\end{array}$ & $\begin{array}{l}1707 \\
1233\end{array}$ & $\begin{array}{r}9 \\
11\end{array}$ & $\begin{array}{l}5.3 \\
8.9\end{array}$ & $\begin{array}{l}1 \dagger \\
1.7\end{array}$ & $(0.7,4.1)$ & 0.239 & $\begin{array}{l}1 \dagger \\
1.5\end{array}$ & $(0.6,3,6)$ & 0.391 \\
\hline Age (y) & $\begin{array}{l}0-3 \\
4-7 \\
8-11 \\
12-15\end{array}$ & $\begin{array}{r}303 \\
387 \\
933 \\
1317\end{array}$ & $\begin{array}{l}4 \\
2 \\
8 \\
6\end{array}$ & $\begin{array}{r}13.2 \\
5.2 \\
8.6 \\
4.6\end{array}$ & $\begin{array}{l}2.9 \\
1.1 \\
1.9 \\
1 \dagger\end{array}$ & $\begin{array}{l}(0.7,5.5) \\
(0.2,5.6) \\
(0.8,10.4)\end{array}$ & $\begin{array}{l}0.373 \ddagger \\
0.175 \S\end{array}$ & $\begin{array}{l}1.5 \\
0.7 \\
1.5 \\
1 \dagger\end{array}$ & $\begin{array}{l}(0.5,4.4) \\
(0.1,3.9) \\
(0.4,5.9)\end{array}$ & $\begin{array}{l}0.738 \ddagger \\
0.791 \S\end{array}$ \\
\hline $\begin{array}{l}\text { Old or newly } \\
\text { diagnosed }\end{array}$ & $\begin{array}{l}\text { Old } \\
\text { New }\end{array}$ & $\begin{array}{l}1845 \\
1095\end{array}$ & $\begin{array}{r}7 \\
13\end{array}$ & $\begin{array}{r}3.8 \\
11.9\end{array}$ & $\begin{array}{l}1 \dagger \\
3.2\end{array}$ & $(1.3,7.9)$ & 0.012 & $\begin{array}{l}1 \dagger \\
2.9\end{array}$ & $(1.1,8.0)$ & 0.039 \\
\hline \multirow[t]{4}{*}{ Season } & $\begin{array}{l}\text { Spring } \\
\text { (Mar-May) }\end{array}$ & 661 & 5 & 7.6 & $1 \dagger$ & & & $1 \dagger$ & & \\
\hline & $\begin{array}{l}\text { Summer } \\
\text { (Jun-Aug) }\end{array}$ & 666 & 3 & 4.5 & 0.6 & $(0.1,2.5)$ & $0.824 \ddagger$ & 0.6 & $(0.1,2.3)$ & $0.786 \ddagger$ \\
\hline & $\begin{array}{l}\text { Autumn } \\
\text { (Sep-Nov) }\end{array}$ & 766 & 5 & 6.5 & 0.9 & $(0.2,3.0)$ & & 0.9 & $(0.2,3.0)$ & \\
\hline & $\begin{array}{l}\text { Winter } \\
\text { (Dec-Feb) }\end{array}$ & 847 & 7 & 8.3 & 1.1 & $(0.3,3.5)$ & & 1.1 & $(0.3,3.4)$ & \\
\hline Total & & 2940 & 20 & 6.8 & & & & & & \\
\hline
\end{tabular}

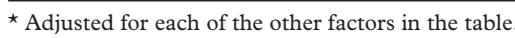

† Reference or baseline category.

†Alternative hypothesis heterogeneity in odds ratios across different levels of factor.

\Alternative hypothesis linear trend in odds ratios across different levels of factor.

The denominator (DKA episodes) for these calculations was determined independently of the ascertainment of cases. The definition of DKA used in this ascertainment was broad, so as to include all episodes, even of moderate severity, in hospitals where it may not be routine to carry out arterial blood gas analysis. It was felt that the cooperation with the study would not have been so high if biochemical details were requested for every episode. Furthermore, cerebral oedema has been reported even with relatively mild acidosis, ${ }^{3}$ so that we were concerned to ensure that we were notified of all possible episodes of DKA where there may have been a risk of cerebral oedema.

The numerator (cerebral oedema) was determined through the BPSU where ascertainment has been previously evaluated. The wide definition for the reporting of cases of cerebral oedema was necessary in order that we would be informed of all possible cases. However, the definition of cerebral oedema was confined to those where other features of raised intracranial pressure were also present. There have been reports that subclinical brain swelling on CT scans may be common during the treatment of DKA, ${ }^{16-18}$ and therefore mild brain swelling on CT scan was not considered confirmatory of cerebral oedema in our study. CT scans were rarely available in children with deterioration in conscious level where there were no other signs of raised intracranial pressure, and these children were excluded from analysis.

Previous studies of the risk of cerebral oedema in DKA in the USA have reported a risk of around $1 \%$ (two major cerebral complications during 214 moderate and severe episodes of $\mathrm{DKA}^{10}$; seven cases of cerebral oedema during 1006 episodes of $\mathrm{DKA}^{4}$ ). A preponderance of newly diagnosed diabetes was reported in the series of Bello and Sotos, ${ }^{4}$ where the risk of cerebral oedema was $3.3 \%$ in new diabetics and $0.23 \%$ in known patients. Similarly, in the largest case series of cerebral oedema reported, ${ }^{3} 60 \%$ of the 69 collected cases occurred in newly diagnosed children. Our study also indicates an increased risk in new compared with established cases of DKA. Interestingly, the overall risk of cerebral oedema complicating DKA in our subjects (1996-1998) was similar to that reported by Bello and Sotos (1975-1990), ${ }^{4}$ suggesting the risk may have been stable over the past 20 years.

The cause or causes of cerebral oedema remain unknown. The use of hypotonic fluids during resuscitation has been suggested as a cause, ${ }^{10}{ }^{19}$ as have reductions in plasma sodium concentration, ${ }^{10-21}$ and rapid changes in plasma osmolality. ${ }^{418}$ Interestingly, there are features of the fluid management of DKA which have until recently differed between the United States and the UK. Specifically, it has been widespread practice to use colloid solutions for initial resuscitation of children with DKA in the UK, but normal or even half normal saline solutions in the USA. Similarly, subsequent rehydration was generally with isotonic saline in the UK but hypotonic saline in the USA. Thus the similar risk of cerebral oedema in the UK and USA suggests that the type of fluid regimen used in the treatment of DKA may have only a limited impact on cerebral oedema risk.

There has been huge variation in reported mortality rates from cerebral oedema. These have ranged from as high as $90 \%{ }^{2}$ to $76 \%,{ }^{8}$ $67 \%,{ }^{5} 64 \%,{ }^{3}$ and $30 \% .{ }^{22}$ Our mortality rate of $24 \%$ is the lowest thus far reported. Only those who fulfilled a strict definition of cerebral oedema were included in our study, and in the majority $(65 \%)$ of cases confirmation was obtained by CT scan. Thus it is unlikely that the difference in mortality rates is related to the definition of cerebral oedema. It is more likely 
that our lower mortality rate reflects our ability to ascertain all cases within the population, rather than selected samples attending a single hospital, or cases reported to authors of case series.

Our data indicate that the neurological morbidity among survivors of cerebral oedema remains significant, $35 \%$ having severe disabilities. This proportion of survivors with disability is similar to the large case series reported by Rosenbloom, ${ }^{3}$ where around $60 \%$ of survivors were neurologically normal. In an earlier case series, only three of ten survivors were free of neurological sequelae. ${ }^{8}$ Differences in morbidity from cerebral oedema may relate to the management of suspected episodes, or may be caused by artefact or bias in the ascertainment of cases in previous series. Current recommended management includes the use of intravenous mannitol to reduce intracranial pressure, which was reported as early as $1970 .{ }^{23} \mathrm{It}$ has more recently been appreciated that for maximum effect, mannitol must be given within five or ten minutes of the initial deterioration in neurological function. ${ }^{24} 25 \mathrm{~A}$ more favourable outcome may be expected with intracranial pressure monitoring and hyperventilation, in addition to mannitol, where this is initiated as soon as cerebral oedema is suspected. ${ }^{26-28}$ However, such preventative measures may not always be effective as only half of the patients in our series (as in that of Rosenbloom $^{3}$ ) had diagnostic neurological change before respiratory arrest; once cerebral herniation has occurred, none of these therapeutic manoeuvres will be beneficial. ${ }^{324}$ We observed a very high morbidity and mortality in subjects presenting initially with respiratory arrest.

In conclusion, cerebral oedema occurs in 6.8 per 1000 episodes of DKA in children under the age of 16 in the UK, and it is more likely to occur in children with newly diagnosed diabetes. It remains a major complication of DKA with a mortality rate of $25 \%$, and $35 \%$ of survivors suffering severe neurological sequelae. Respiratory arrest is an important predictor of outcome as all of those presenting in this way died or were left with significant morbidity. The cause of cerebral oedema complicating DKA is still unknown, and at present, earlier recognition and prevention of DKA, particularly in young children with newly diagnosed diabetes, may be the only way of reducing the associated morbidity and mortality. We are currently carrying out the first population based case control study to determine factors in the presentation and clinical course which may be related to the development of cerebral oedema.

We are extremely grateful to Claire Garbett, who coordinated the Oxford based reporting system and retrieved all of the missing data so efficiently. We are indebted to all the paediatricians who have been so generous with their time and effort in providing us with the necessary information, and to Richard Lynn and the staff at the BPSU for their help and advice. The British Diabetic Association funded the study.

1 Dillon ES, Riggs HE, Dyer Ww. Cerebral lesions in uncomplicated fatal diabetic acidosis. $\mathrm{Am}{ }_{7} \mathrm{Med} \mathrm{Sc}_{\mathrm{c}}$ plicated fatal
2 Hammond P, Wallis S. Cerebral oedema in diabetic ketoacidosis. Still puzzling-and often fatal. BMf 1992;305. dosis.

3 Rosenbloom AL. Intracerebral crises during treatment of diabetic ketoacidosis. Diabetes Care 1990;13:22-33.

4 Bello FA, Sotos JF. Cerebral oedema in diabetic ketoacidosis in children. Lancet 1990;336:64.

5 Mel JM, Werther GA. Incidence and outcome of diabetic cerebral oedema in childhood: are there predictors? Paediatr Child Health 1995;31:17-20.

6 Edge JA, Dunger DB. Causes of death in children and young people with diabetes mellitus. Diabet Med 1997; 15(suppl 1):57.

7 Edge JA, Ford-Adams ME, Dunger DB. Causes of death in children with type 1 diabetes, 1990-1996. Arch Dis Child 1999;81:318-23.

8 Duck SC, Wyatt DT. Factors associated with brain herniation in the treatment of diabetic ketoacidosis. F Pediatr 1988;113:10-14.

9 Tubiana-Rufi N, Thizon-de-Gaulle I, Czernichow P. Hypothalamopituitary deficiency and precocious puberty following hyperhydration in diabetic ketoacidosis. Horm Res 1992;37:60-3.

10 Harris GA, Fiordalisi I, Harris WL, et al. Minimizing the risk of brain herniation during treatment of diabetic ketoacidaemia: a retrospective and prospective study. $\mathcal{F}$ Paediatr 1990;117:22-31

1 Hall SM, Glickman M. Report from the British Paediatric Surveillance Unit. Arch Dis Child 1990;65:807-9.

12 Haines LC, Swift PGF. Report of the 1994 BPA/BDA survey of services for children with diabetes: changing patterns of care. Diabet Med 1997;14:693-7.

13 British Paediatric Association. British Paediatric Association handbook 1995-1996. Northampton: Land \& Unwin, 1995.

14 NAESC. Hospitals with child admissions. In: Education for sick children; directory of current provision in England and Wales. London: National Association for the Education of Sick Children, 1994:163-87.

15 Pinkney JH, Bingley PJ, Sawtell PA, et al. Presentation and progress of childhood diabetes mellitus: a prospective population-based study. Diabetologia 1994;37:70-4

16 Krane EJ, Rockoff MA, Wallman JK, Wolfsdorf JI. Subclinical brain swelling in children during treatment of diabetic ketoacidosis. N Engl f Med 1985;312:1147-51.

17 Hoffman WH, Steinhart CM, El Gammal T, et al. Cranial $\mathrm{CT}$ in children and adolescents with diabetic ketoacidosis. Am $\mathcal{F}$ Neuroradiol 1988;9:733-9.

18 Durr JA, Hoffman WH, Sklar AH, et al. Correlates of brain edema in uncontrolled IDDM. Diabetes 1992;41:627-32.

19 Harris GA, Fiordalisi I. Physiologic management of diabetic ketoacidemia. A 5-year prospective pediatric experience in 231 episodes. Arch Pediatr Adolesc Med 1994;148:1046-52.

20 Silver SM, Clark EC, Schroeder BM, Sterns RH. Pathogenesis of cerebral edema after treatment of diabetic ketoacidosis. Kidney Int 1997;51:1237-44.

21 Hale PM, Rezvani I, Braunstein AW, et al. Factors predicting cerebral edema in young children with diabetic ketoacidosis and new onset type I diabetes. Acta Paediatr 1997;86: sis and $626-31$.

22 Levitsky L, Ekwo E, Goselink CA, et al. Death from diabetes DM) in hospitalised children (1970-1988). Pediatr Res 1991;29:195A.

23 Metzger AL, Rubenstein AH. Reversible cerebral edema complicating diabetic ketoacidosis. Diabetes 1970;2:108-11. 4 Duck SC, Kohler E. Cerebral edema in diabetic ketoacidosis [letter]. F Pediatr 1981;98:674.

25 Franklin B, Liu J, Ginsberg-Fellner F. Cerebral edema and ophthalmoplegia reversed by mannitol in a new case of insulin-dependent diabetes mellitus. Pediatrics 1982;69:8790.

26 McAloon J, Carson D, Crean P. Cerebral oedema complicating diabetic ketoacidosis. Acta Paediatr Scand 1990;79:115-17.

27 Greene SA, Jefferson IG, Baum JD. Cerebral oedema complicating diabetic ketoacidosis. Dev Med Child Neurol 1990; 32:633-44.

28 Wood EG, Go-Wingkun J, Luisiri A, Aceto T. Symptomatic cerebral swelling complicating diabetic ketoacidosis documented by intraventricular pressure monitoring: survival mented by intraventricular pressure monitoring: survival without

\section{Commentary}

DIABETIC KETOACIDOSIS IN THE YOUNG: HOW DANGEROUS IS IT?

An episode of diabetic ketoacidosis (DKA) in a young person with diabetes is a medical emergency, and frequently causes great anxiety in the medical and nursing staff treating the condition. Despite the plethora of guidelines and protocols, all with meticulous details of fluid and electrolyte therapy and insulin replacement, there is sometimes a feeling of crisis about the management of the patient, and the well laid plans of fluid balance and blood glucose and $\mathrm{pH}$ monitoring may often need to be rewritten during the period of treatment. 
The spectre of a sudden collapse and deterioration in the patient colours the medical management, with a major concern that the complication of cerebral oedema will occur if there are problems during the treatment. How appropriate is that concern, what increases the risk of cerebral oedema, and what is the outcome of this complication of DKA?

Edge and her colleagues from Oxford have gone a long way towards answering these questions. In their meticulous study, they present original prospective data for the UK. They used the combined forces of the British Paediatric Surveillance Unit of the Royal College of Paediatrics and Child Health, Diabetes UK, and the British Society of Paediatric Endocrinology and Diabetes to identify cases of cerebral oedema. During the same time span, they identified cases of DKA presenting over a two year period to hospitals throughout the UK. Data was obtained on nearly 6000 episodes of DKA over two years with 225 consultants reporting details of the patients. The research group, the professional organisations and the paediatricians are to be congratulated on their efforts in collecting this unique population data.

Previously published retrospective data reported that $70-80 \%$ of deaths in young people $<12$ years old are from the complication of cerebral oedema in the presentation and management of DKA. Edge's prospective study suggests that in the recent years mortality has dropped to $24 \%$ but that morbidity in the survivors is high at $35 \%$. Respiratory arrest results either in death $(80 \%)$ or severe handicap in the survivors. It is possible that the group's lower mortality rate was influenced by their wide criteria of DKA and their clinical definition of cerebral oedema. Previous studies have shown radiological evidence of brain oedema without any of the symptoms of a dissociation syndrome and/or cerebral herniation. ${ }^{1}$ Indeed asymptomatic cerebral oedema may be present in all cases of DKA, and therefore, as suggested by the Oxford Group, any change in signs and symptoms during the presentation and management of DKA is important. The data suggest that recently paediatricians have reacted to neurological change and deterioration in DKA with an intensive care approach. However, while mortality is improving, it is still high ( 1 in 4 cases of cerebral oedema) and long term handicap is a major threat. A concern from the study is that DKA patients are managed without immediate access to paediatric intensive care facilities and/or that clinical teams are not reacting to early subtle changes in neurological signs. It is too late to react at the time of a respiratory arrest.

The overall risk for cerebral oedema per episode of DKA is low $(0.7 \%)$. Of concern is the threefold risk of cerebral oedema in new patients of type 1 diabetes mellitus who present in DKA. The assumption here is that the risk factor is the length of time the new patient is exposed to severe metabolic derangement. Although this may be related to failure to contact medical advice, the worry is that it may reflect failure by general practitioners to diagnose early diabetes and/or ketoacidosis in young people. A reduction in the rate of DKA at presentation of diabetes is a target for all clinical teams involved in diabetes in the young. ${ }^{23}$ Surely the rate should be $0 \%$.

This study shows that DKA is unfortunately relatively common: on average there are 3000 admissions per year (averaging over 50 admissions per week) to hospitals throughout the UK. Although $99 \%$ of patients recover completely, this represents a major health problem that demands considerable resources from the NHS. Prevention must be the best approach and all centres involved in young people with diabetes must look at reducing their local DKA rate significantly.

The data on DKA confirms the concern over young girls with brittle diabetes. ${ }^{45}$ Five percent of cases accounted for nearly one quarter of admissions for DKA across the UK. We can empathise with this situation, as we all have one or more patients with brittle diabetes in our clinic at any time, who demand an enormous efforts from all of the staff. The basis of the DKA is almost certainly chronic insulin insufficiency secondary to poor adherence to insulin regimens. ${ }^{6}$ Innovative methods are required to support these young people and to prevent their admission to hospital with DKA. ${ }^{5}$ These are likely to be based around support systems in addition to standard UK clinics, that are designed to promote adherence to treatment: they will require different resources and are likely to use approaches from psychology and social science. Evidence of their effectiveness will need to be evaluated.

This study did not throw any further light on the cause of cerebral oedema in DKA. Specifically, the authors could not relate the neurological deterioration to any particular fluid or insulin therapy. Their suggestion that the overall risk is unchanged suggests that more detailed fluid balance and insulin therapy for DKA fails to prevent cerebral oedema. Their prospective case control study may shed more light on this area. Interestingly, very recent data from the USA during the mid 1990s suggest that bicarbonate therapy is a major predictor of cerebral oedema. ${ }^{7} \mathrm{UK}$ practice differs and it would be surprising if bicarbonate were a factor; bicarbonate is nearly always omitted from the guideline recommendations of DKA treatment in the UK. Judicious use of insulin and fluid replacement appears to be sound clinical advice and the British Society for Paediatric Endocrinology and Diabetes DKA Guidelines should be the recommendation on all wards throughout the UK.

DKA in the young is a dangerous condition, but thankfully the majority of patients receive excellent care in the UK and recover completely. Perhaps of more concern from this study is the persisting high background level of DKA in our population of young people with diabetes. Novel strategies are required to reduce the incidence of DKA.

\section{STEPHEN GREENE}

Tayside Institute of Child Health,

Ninewells Hospital and Medical School,

Dundee DD1 9SY, UK 
1 Krane EJ, Rockoff MA, Wallman JK, et al. Subclinical brain swelling in children during treatment of diabetic ketoacidoswelling in children during treatment of
sis. New Eng $\mathcal{7}$ Med 1985;312:1147-51.

Sis. New Eng 7 Med 1985;312:1147-51.
2 ISPAD consensus guidelines for the management of diabetes melISPAD consensus guidelines for the management of diabetes mel-
litus in children and adolescents. The Netherlands: ISPAD litus in children and adolescents. The
Medical Forum International, 2000.

3 Scottish Intercollegiate Guideline Network. Report on good practice in the care of children and young people with diabetes. SIGN Guideline 10. Edinburgh: Royal College of Physicians, 1996.

4 Greene S, Morris A. The management of brittle diabetes mellitus. Current Paediatrics 1999;9;247-51.
5 Greene SA. Is even moderate control of diabetes in adolescence feasible? In Gill G, Pickup J, Williams G, eds. Difficult Diabetes. Oxford: Blackwell Science, 2001.

6 Morris AD, Boyle DIR, McMahon AD, et al for the DARTS/MEMO collaboration. Adherence to insulin treatment, glycaemic control and ketoacidosis in IDDM. Lancet 1997:350;1505-10.

7 Glaser N, Barnett P, McCaslin I, et al. Risk factors for cerebral edema in children with diabetic ketoacidosis. New Eng $\mathcal{F}$ Med 2001;344:264-9.

\section{Hiding place for mycobacteria}

I suspect that in many of our minds "the literature" begins at about the time we entered medical school. This may, of course, mean that much useful research is ignored. A fascinating example of the value of knowing about findings from a previous era has been provided by work on the location of latent tubercle bacilli (R Hernández-Pando and colleagues. Lancet 2000;356:2133-8).

In 1927, E L Opie and J D Aronson reported transmitting tuberculosis to guinea pigs from less than $10 \%$ of old tuberculous lung lesions but from almost $50 \%$ of specimens of apparently unaffected lung, suggesting that the bacilli in latent tuberculosis might live in normal lung tissue rather than, as is often supposed, in old, encapsulated lesions. The recent Lancet report has described similar findings using DNA technology.

They used in situ and conventional PCR to examine apparently normal postmortem lung tissue from one experimental and two control groups: 47 people who had died in tuberculosis endemic areas (13 in Ethiopia and 34 in Mexico) but had not had tuberculosis (experimental group), six who had died in Norway and had not had tuberculosis (negative controls), and six who had died in Ethiopia of tuberculosis (positive controls). All of the negative controls proved negative and all of the positive controls positive on PCR testing of macroscopically normal lung tissue for $M$ tuberculosis DNA. In the experimental group, however, the tests were positive in 15 cases (five of 13 in Ethiopia, and 10 of 34 in Mexico). (Eight of the Mexican subjects were children of 16 years or less and six of those had positive PCR tests.) None of the tissue samples showed histological evidence of tuberculosis. The mycobacterial DNA was located within alveolar and interstitial macrophages, type II pneumocytes, endothelial cells, and fibroblasts.

The presence of mycobacterial DNA does not, of course, prove the presence of living organisms and the method used would detect BCG DNA as well as that from infective $M$ tuberculosis (see commentary, Ibid:2113-4). Nevertheless, if latent mycobacteria do reside in normal lung cells, rather than in old encapsulated lesions, fresh thoughts about the elimination of latent infection may be needed.

ARCHIVIST 